\title{
Loneliness Recognition Based on Mobile Phone Data
}

\author{
Zhongqiu $\mathrm{Li}^{1, \mathrm{a}}$, Dianxi Shi ${ }^{2, \mathrm{~b}}$, Feng Wang ${ }^{3, \mathrm{c}}$ and Fan Liu ${ }^{4, \mathrm{~d}}$ \\ ${ }^{1,2,3,4}$ Nation Laboratory for Parallel and Distributed Processing, School of Computer, \\ National University of Defense Technology, Changsha 410073, China \\ alizhongqiu267@163.com, bdxshi@nudt.edu.cn, cwangfengfighting@gmail.com, \\ d412316212@qq.com
}

\begin{abstract}
Keywords: loneliness recognition, smartphone, machine learning, mental health
Abstract. Nowadays, the definition of health is not only the absence of disease, but both physical and mental health. Loneliness as an important measure of mental health has become a topic that can not be ignored. In this paper, we study the problem about loneliness of individuals can be automatically recognized using mobile phone data (app usage data, call log, SMS, GPS data, Bluetooth proximity data and so on). In our study, we used 46 participants' data, divided them into risk and non-risk group based on self-reported scales for loneliness. We then compared the two groups to analyze the differences of mobile phone usage. And then we selected four kinds of classifiers - Support Vector Machine (SVM), Random Forest, Neural Network, and Gradient Tree Boosting (GTB) - to recognize loneliness automatically based on mobile phone data. The result showed that Random Forest can obtain the best performance with the accuracy of $70.68 \%$ for a 2 -class loneliness recognition problem.
\end{abstract}

\section{Introduction}

With the development of economy and technology, the popularity of mobile phone has been increasing rapidly in recent years. The mobile phone has now become an indispensable tool in people's daily life, which is no longer a simple communication tool, but provides many functions, such as surfing the Internet, playing games, taking photos, contacting with others and entertainment. As the social psychologist Miller wrote "The Smartphone Psychology Manifesto" [1], the smartphone has become a new research tool for psychology. Researchers have found the close relationship between mobile phone and mental health. According to previous researches, mobile phone data can be used in many fields, such as mood assessment [2,3], stress recognition [4,5], happiness recognition [6], bored detection [7], and so on.

Especially in recent, mental health has become a hot topic in human's daily life. The definition of health is not only the absence of disease, but both physical and mental health. Loneliness as an important metric of mental health, especially come into people's notice. It is generally known that behavior data has a great help for us to understand mental disorder, because mental disorder will change the behavior of the individual performance. Similarly, we can judge from the individual's behavior to their mental health, and smartphone can exactly sense to the individual's behavior.

At the right moment, the growth of mobile phones provides us with a new way to recognize loneliness. Today's mobile phones are equipped with rich sensors, such as gyroscope, accelerator, magnetometer, light sensor, microphone, etc. In addition to such sensor data, mobile phone can generate a lot of usage data - call records and SMS records, Bluetooth proximity data, WiFi data, and application usage data - which can directly response user's daily behavior.

In this paper, we proposed a novel approach for loneliness recognition using mobile phone data, without any medical equipment so that drastically reduced the cost. First, we extracted the subset from a public dataset called StudentLife study. Second, we divided all participants into two groups based on self-report: a risk group (whose scores indicated a tendency of loneliness) and a non-risk group, and then compared the differences between risk group and non-risk group. Last, we took the method of data future fusion, used four kinds of classifiers - Support Vector Machine (SVM), Random Forest, Neural Network, and Gradient Tree Boosting (GTB). The final classification result of accuracy can achieve $70.68 \%$. 
The rest of this paper is organized as follows. Section 2 briefly introduces the related work and background. Section 3 shows the dataset of this project. Section 4 and Section 5 describe the methodology and experiment result of the research. Section 6 and Section 7 introduce the conclusion and future work of this research.

\section{Background}

The recognition of loneliness using mobile phone data has not become the main target of the research community so far. The main goal of our work is to recognize user's loneliness based on mobile phone data. In this section, we will introduce the relevant background from two domains - loneliness assessment and mobile phone sensing.

Loneliness and loneliness assessment. The word "loneliness" comes from the medical field, originally used to indicate dysfunction in interpersonal communication and emotional expressions. Loneliness and loneliness assessment is a research emphasis in the field of psychology. Perlman and Peplau (1981) pointed out that loneliness referred to individuals experience negative emotions due to lack of social networking [8]. According to Lopata (1969), Loneliness is an emotion which people's current social situation does not meet their aspiration [9]. According to Masi, Chen, Hawkley, \& Cacioppo (2011), an individual may be lonely in a crowd or socially contented while alone [10]. Loneliness has now become a universal human experience and related to many factors, such as stress $[11,12]$, depression [12,13], shyness [14], Internet addiction [15,16], social anxiety [17], and mobile phone addiction $[17,18,19]$. Before this, loneliness is always measured by psychological scales, such as UCLA Loneliness Scale [20], Autism Behavior Checklist [21], Clancy Behavior Scale [22], etc. But the disadvantages of scales is obvious, because it has a great deal of subjectivity, leading to a inaccurate result.

Mobile phone sensing. Previous works tried to focus on many fields using mobile phone data, such as mood assessment, stress recognition, academic performance, daily happiness recognition and so on. LiKamWa et al. [2], build a mood sensor called "MoodScope" to collect six pieces of usage information, that is SMS, E-mail, phone call, web browsing, apple usage, and location, to infer user's mood by a multi-linear regression algorithm. Andrey Bogomolov et al. [4,6], used mobile phone data (including phone call, SMS, and Bluetooth ), weather conditions and individual traits ,using multiple methods of classifier, such as Random Forest, Neural Network, SVM, GBM, to recognize user's daily stress and daily happiness. Wang et al. [23], collected the mobile sensor data of about 48 students in Dartmouth Colleague for a period of 10 weeks to analysis the relationship between the stress, sleep ,activity, mood, sociability, mental health and academic performance. N. Eagle, et al. [24] used data from mobile phones to explore social and individual patterns in society. MIT media lab have developed a sensor framework named Funf [25] used for mobile devices and data processing. The basic idea of Funf is to install a collector on a mobile phone, to track a group of users of all kinds of activities, which can be analyzed user behavior patterns.

\section{Dataset}

In this paper, we adopted a subset of the StudentLife dataset [23] to recognize loneliness. The StudentLife dataset is a large, longitudinal dataset which is from 48 students at Dartmouth over the 10 -week term. It includes 53 GB of continuous data and contains four types of data, namely, sensing data, EMA data, survey responses and educational data. Sensor data includes: a) objective sensing data (sleep, conversation duration, conversation frequency and physical activity); b) location-based data (location, co-location, indoor/outdoor mobility and distance covered); c) other phone data (Bluetooth, WiFi, audio screen lock/unlock, light, phone charge and app usage). Survey data contains the daily self-reports, including: UCLA Loneliness Scale measuring Loneliness level, flourishing scale measuring flourishing level, perceived stress scale (PSS) measuring stress level, patient health questionnaire (PHQ -9) measuring depression level, and the Big Five measuring personal traits. 
Students have different activities at different times, so that in our dataset we divided a 24 hour day into 3 epochs - day epoch (9am - 6pm), evening epoch (6pm - 12am), night epoch (12am - 9am). Next we will discuss the behavior pattern differences in the different epoch.

Table 1 summarized the subset of StudentLife dataset we used. In particular, phone data contains phone calls, SMS, Bluetooth, WiFi, app usage, phone charge, screen lock/unlock. Big Five personality trait is a 44-question questionnaire using 1-5 point developed by John et al. [26].

Table1 Subset of StudentLife dataset

\begin{tabular}{ll}
\hline \multirow{3}{*}{ Personality } & Openness \\
& Consciousness \\
& Extraversion \\
& Agreeableness \\
& Neuroticism \\
\hline \multirow{3}{*}{ Mental Health } & PHQ -9(pre and post ) \\
& UCLA Loneliness Scale (pre and post ) \\
& Perceived stress scale (pre and post ) \\
\hline \multirow{3}{*}{ Sensing data } & Activity duration \\
& Conversation duration \\
& Conversation frequency \\
& Location (GPS and WiFi) \\
& Sleep duration \\
& Phone data \\
\hline
\end{tabular}

As for loneliness metrics, we used UCLA loneliness scale, a 20-item scale designed to measure one's subjective feelings of loneliness .10 of these items are positive statements and the rest are negative .The assessment results range from 20 to 100, and the higher score mean the higher level of loneliness. When the score is more than 44 suggests there is a clear loneliness. In our dataset, the lowest score is 25 while the highest score is 64 . Fig. 1 shows the Loneliness scores distribution of 46 participants.

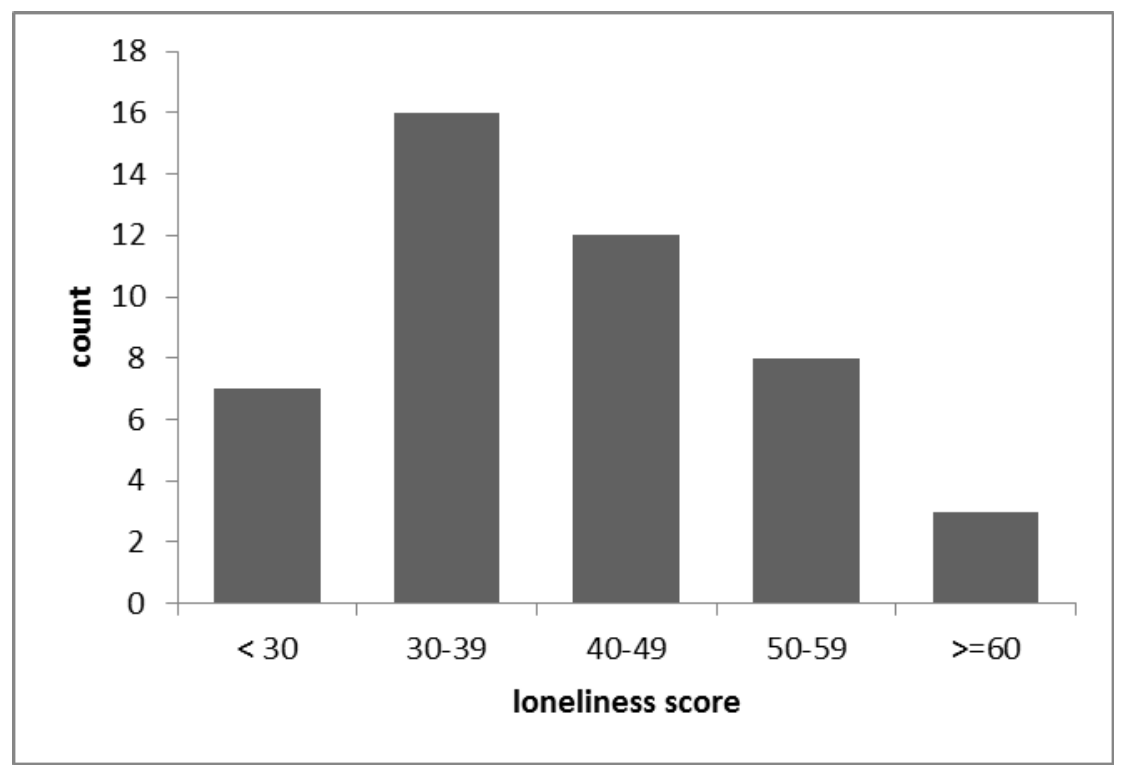

Fig. 1. Loneliness scores distribution

According to the score of loneliness measurement, participants were divided into two groups: a risk group (whose scores show that they feel lonely) and a non-risk group, then we will see the mobile 
phone usage patterns of these two groups are very different. As fig. 2 (a) shows, compared with non-risk group, the risk group spent more time on mobile phone per day (risk group: 7.22 hour vs. non-risk group: 5.71 hour ). Data also shows that in the evening and on weekends, the time of risk group spend on mobile phone is significantly more than non-risk group.

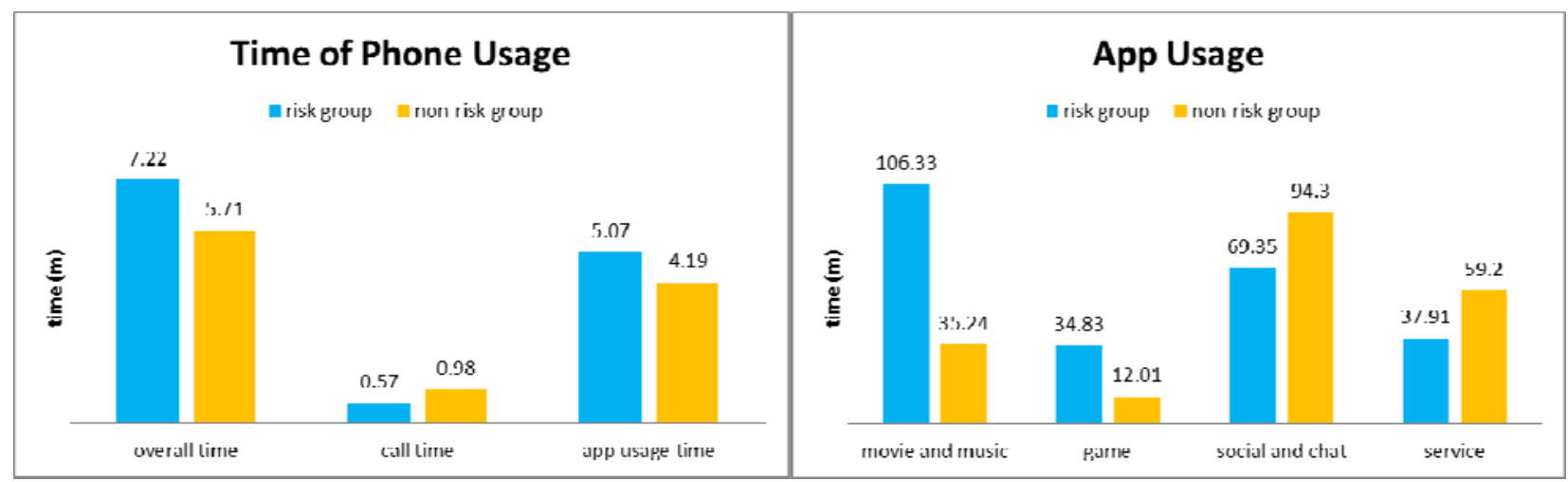

Fig. 2. (a) time of phone usage, (b) app usage

\section{Feature Extraction}

Taking example by the previous works on stress recognition using mobile phone data [4], we derived a basic set of features in four categories.

Concerning app usage, using k-means algorithm to cluster applications into $k$ categories .The manner People use app with different character and deposition will be totally different. For example, outgoing people may tend to use more social interaction apps, while introverted people may use less. Due to the most of applications are download from Google Play, we extracted most application description and category information from Google Play (about 93.76\%). And then we extracted key words into k categories by k-means algorithm. Those categories include work, map navigation, health, social interaction, news and info, media, game, e-commerce, and so on. Table 2 lists the application classification of part of dataset.

Table 2 Classification of App

\begin{tabular}{llll}
\hline Package Name & App name & Keywords & Categories \\
\hline Com.google.android.gm & Gmail & Mail; Efficiency; Work & Work \\
Com.google.earth & Google Earth & Traffic; Navigation; Map & Map navigation \\
Com.rjfittime.app & FitTime & Fitness; Exercise & Health \\
Com.twitter.android & Twitter & Social Network; Chatting & Social Interaction \\
Com.blackboard.android & Mobile Learn & News; Information; Reading & News and Info \\
Com.google.android.youtube & YouTube & Video; Player & Media \\
Com.imangi.templerun2 & templeRun2 & Parkour; Puzzle; Relaxation & Game \\
Com.ebay.mobile & eBay & Shopping;Mall & E-commerce \\
\hline
\end{tabular}

As figure 2 (b) shows, risk group spent more time on entertainment application (movie and music, game, etc.) than non-risk group, because this kind of app is easy to pass the time and there's no need to contact with others. In the same way, non-risk group spent more time on interaction application (social and chat, service, etc.) than risk group, because this kind of app need more interaction with others. The above shows that lonely people are more likely to use applications, but more is no-interaction application, because they tend to not contact with others, even on the Internet.

As for GPS, we extracted 2 features: stay points and entropy. We used a clustering algorithm that presented by Rodriguez and Laio [27] to calculate user's stay points, which help us to find out how many places user has stayed in a day. For entropy calculation, we applied Information Entropy [28], which is explained in Equation (1). 


$$
\mathrm{H}(\mathrm{u})=-\sum_{i}^{n} p_{i} \cdot \log _{2} p_{i} \text {. }
$$

Where $p_{i}$ represents the proportion of the total data for the user in the $i$ position. Information entropy $\mathrm{H}(\mathrm{u})$ is used to measure the amount of information in information theory. That is to say, the more orderly a system is, the lower information entropy is and vice versa. So the information entropy is the metric of the ordering degree. For example, if a user stays at home all day, there is only one position after clustering, then the entropy is 0 , indicating in an orderly state.

Many previous works indicate that social interactions from mobile phone data can predict people's behavior and traits. Such social interactions from mobile phone data include call, SMS, and proximity (Bluetooth scanning).

The interaction features can be divided into 4 categories (1) general usage, (2) active behavior, (3) regularity, (4) diversity.

General phone usage include the total number of calls (incoming calls / outgoing calls / missed calls ), the total number of SMS (received SMS / sent SMS ), the number of Bluetooth IDs, the times most common Bluetooth ID is seen. In addition, we calculated the following ratios: incoming to outgoing calls, missed to all calls, received to sent SMS .

Features for active behavior consist of: percentage of calls during the night, percentage of SMS during the night, SMS response rate, percentage of initiated SMS, and so on.

As for regularity features, we measured the time elapsed between two events, such as calls, SMS, proximity, as well as calls and SMS. In particular, even two users' time between calls or SMS is same, but the time between calls and SMS may be different.

Concerning diversity features, we measured the number of unique contacts called and who called, the number of unique contacts associated with missed calls , the number of unique contacts SMS sent to and received from, contacts to interactions ratio.

Table 3 Basic Features of Interaction Data

\begin{tabular}{l}
\hline General usage \\
\hline Total number of calls (Incoming +Outgoing, incoming, outgoing, missed ) \\
Total number of missed SMS (received, sent ) \\
Total number of Bluetooth IDs \\
Times most common Bluetooth ID is seen \\
\hline Active Behavior \\
\hline percentage of calls/SMS during the night \\
SMS response rate \\
percentage of initiated SMS \\
\hline Regularity \\
\hline Time elapsed between two calls \\
Time elapsed between two SMS \\
Time elapsed between call and SMS \\
Time elapsed between proximities \\
\hline Diversity \\
\hline Number of unique contacts (called and who called ) \\
the number of unique contacts associated with missed calls \\
the number of unique contacts( SMS sent to and received from) \\
contacts to interactions ratio
\end{tabular}

Apart from app usage, GPS, and interaction data, there are some other data, such as phone lock/unlock mode, phone charge, conversation. Conversation represents whether a user is surrounded by a conversation environment, and recorded the beginning and ending time of conversations. 


\section{Methodology and Experiment Result}

In order to find out the relationship between variables, we adopted Pearson correlation analysis where $r$ indicates the direction and strength of correlation, and $p$ indicates the significance of the result. Due to the high dimension of features, we applied principal component analysis (PCA) for dimension reduction.

Table 4 Correlations between sensing data and UCLA loneliness scale

\begin{tabular}{lll}
\hline Sensing data & $\mathrm{r}$ & $\mathrm{p}$-value \\
\hline App frequency & 0.360 & 0.031 \\
App frequency during the day & 0.362 & 0.029 \\
App frequency during the night & 0.341 & 0.047 \\
Bluetooth frequency & -0.150 & 0.233 \\
SMS frequency & -0.300 & 0.051 \\
SMS frequency during evening & -0.320 & 0.078 \\
Call frequency & 0.135 & 0.221 \\
Call frequency during evening & 0.262 & 0.087 \\
Call duration & -0.279 & 0.084 \\
Call duration during evening & -0.355 & 0.042 \\
Indoor mobility for day & -0.341 & 0.045 \\
Activity duration & -0.387 & 0.018 \\
Activity duration for day & -0.315 & 0.044 \\
Activity duration for evening & -0.425 & 0.007 \\
\hline
\end{tabular}

As table 4 shows, we find some correlations between app frequency, SMS frequency, call duration, activity duration, indoor mobility and the UCLA loneliness scale. Loneliness is positively associated $(\mathrm{r}=0.360, \mathrm{p}=0.031)$ with app usage frequency, with no significant time difference. There is no significant relationship $(\mathrm{r}=-0.150, \mathrm{p}=0.233)$ between loneliness and Bluetooth proximities. A weak negative relationship $(\mathrm{r}=-0.300, \mathrm{p}=0.051)$ is between loneliness and SMS, especially during night, and so is call duration $(\mathrm{r}=-0.320, \mathrm{p}=0.078)$. Loneliness has no significant relation $(\mathrm{r}=0.135, \mathrm{p}=$ $0.221)$ with call frequency, but has a positive correlation $(\mathrm{r}=-0.355, \mathrm{p}=0.042)$ during evening. Indoor mobility measures how much a student moved indoor during the day, which has a negative correlation ( $\mathrm{r}=-0.341, \mathrm{p}=0.045)$ with loneliness, so it suggests that inactive students are more easily feel lonely. We also can see activity duration and loneliness have a significant negative correlation $(\mathrm{r}$ $=-0.387, \mathrm{p}=0.018$ ), whether it is day or night.

In order to solve the final goal - the classification problem, we trained several classifiers: Random Forest, Support Vector Machine (SVM), Neural Network, and Gradient Tree Boosting (GTB). We formulated the recognition of loneliness as a classification problem with 2 classes (not lonely and lonely). We choose the personal report as the ground truth data. According to the score of UCLA loneliness scale, we divided all the users into two classes. The ground truth label 0 means "lonely" and label 1 means "not lonely". We divided all the data into training set and test set with the proportion of 80: 20. It turned out that Random Forest has the best performance of classification. In order to assess the performance of classifier, we applied metrics of accuracy, precision, recall, and F-score. Table 5 shows the performance of four classifiers.

Table 5 Performance of classifiers

\begin{tabular}{lllll}
\hline Classifier & Accuracy & Precision & Recall & F-score \\
\hline Random Forest & $68.67 \%$ & $70.68 \%$ & $67.89 \%$ & $66.54 \%$ \\
SVM & $59.22 \%$ & $62.34 \%$ & $61.71 \%$ & $64.88 \%$ \\
Neural network & $53.15 \%$ & $54.91 \%$ & $56.34 \%$ & $58.94 \%$ \\
GTB & $60.57 \%$ & $65.82 \%$ & $61.29 \%$ & $57.30 \%$ \\
\hline
\end{tabular}


Moreover, we ran experiment with 3 classes ("not lonely", "neutral", and "lonely"), with the label -1 for "not lonely", label 0 for "neutral", and label 1 for "lonely". But as we expected, the classification is not so good with the highest accuracy is $43.91 \%$.

\section{Conclusion}

The main goal of this paper is to prove that loneliness can be recognized from mobile phone data. To achieve this goal, we collected two different sets of data: mobile phone data and personal self-reports. We handled the problem as a 2-class problem.

All in all, we believe that our result ( $70.68 \%$ for a 2-class classification problem) has fully proved that individual loneliness can be automatically recognized from mobile phone. For future work, we will improve the accuracy of recognition and then put forward a personal assessment model that can adapt recognize user's loneliness, so that we can identify the levels of loneliness in a real-time way.

\section{Acknowledgement}

This work was supported by the National Nature Science Foundation of China (No.61202117).

\section{References}

[1] Miller G. The Smartphone Psychology Manifesto.[J]. Perspectives on Psychological Science, 2012, 7(3):221-237.

[2] Likamwa R, Liu Y, Lane N D, et al. MoodScope: building a mood sensor from smartphone usage patterns[C]// Proceeding of the 11th annual international conference on Mobile systems, applications, and services. ACM, 2013:389-402.

[3] Ma Y, Xu B, Bai Y, et al. Daily Mood Assessment Based on Mobile Phone Sensing[C]// Wearable and Implantable Body Sensor Networks (BSN), 2012 Ninth International Conference on. IEEE, 2012:142-147.

[4] Bogomolov A, Lepri B, Ferron M, et al. Daily Stress Recognition from Mobile Phone Data, Weather Conditions and Individual Traits[J]. Eprint Arxiv, 2014:477-486.

[5] Lu H, Frauendorfer D, Rabbi M, et al. StressSense: Detecting Stress in Unconstrained Acoustic Environments using Smartphones[C]// Proceedings of the 2012 ACM Conference on Ubiquitous Computing. 2012:351-360.

[6] Bogomolov A, Lepri B, Pianesi F. Happiness Recognition from Mobile Phone Data[C]// Social Computing / IEEE International Conference on Privacy, Security, Risk and Trust, 2010 IEEE International Conference on. IEEE, 2013:790-795.

[7] Pielot M, Dingler T, Pedro J S, et al. When attention is not scarce-detecting boredom from mobile phone usage[C]/Proceedings of the 2015 ACM International Joint Conference on Pervasive and Ubiquitous Computing. ACM, 2015: 825-836.

[8] Perlman, D., \& Peplau, L. A. (1981). Toward a social psychology of loneliness. In S.Duck \& R. Gilmour (Eds.). Personal relationships (Vol.3, pp. 31-56). New York:Academic Press.

[9] Lopata, H. Z. (1969). Loneliness: Forms and components. Social Problems, 17, 248-262

[10]Masi, C. M., Chen, H. Y., Hawkley, L. C., \& Cacioppo, J. T. (2011). A meta-analysis of interventions to reduce loneliness. Personality andSocial Psychology Review, 15(3), 219-266.

[11] Yaacob, S., Juhari, R., Talib, M., \& Uba, I. (2009). Loneliness, stress, self esteem and depression among Malaysian adolescents. Jurnal Kemanusiaan Bil. 14, Dis.85-95. 
[12] Wang J J, Snyder M, Kaas M. Stress, loneliness, and depression in Taiwanese rural community-dwelling elders[J]. International Journal of Nursing Studies, 2001, 38(3):339-47.

[13] Adams K B, Sanders S ,, Auth E A. Loneliness and depression in independent living retirement communities: risk and resilience factors.[J]. Aging \& Mental Health, 2004, 8(6):475-485.

[14]Bian M, Leung L. Linking Loneliness, Shyness, Smartphone Addiction Symptoms, and Patterns of Smartphone Use to Social Capital[J]. Social Science Computer Review, 2014, 33(1):61-79.

[15]Özdemir Y, Kuzucu Y, Şerife Ak. Depression, loneliness and Internet addiction: How important is low self-control?[J]. Computers in Human Behavior, 2014, 34(4):284-290.

[16]Zarbakhsh-Bahri M R, Rashedi V, Khademi M J. Loneliness and Internet addiction in students[J]. Journal of Health Promotion Management, 2013, 2(1):32-38.

[17]Fletcher K. Smartphone Addiction in Relation with Social Anxiety and Loneliness Among University Students in Turkey[J]. European Psychiatry, 2015, 30:505-505.

[18]Liu H, Wang H L, Department E M, et al. Mobile phone addiction tendency and loneliness in college students[J]. Chinese Mental Health Journal, 2012, 26(1):66-69.

[19]Çetin Tan, Pamuk M, Dönder A. Loneliness and Mobile Phone[J]. Procedia - Social and Behavioral Sciences, 2013, 103:606-611.

[20]Russell D , Peplau L A, Cutrona C E. The revised UCLA Loneliness Scale: concurrent and discriminant validity evidence.[J]. Journal of Personality \& Social Psychology, 1980, 39(3):472-480.

[21]Cassidy A. Autism Behavior Checklist[M]. Springer New York, 2013.

[22] Warren Z, Dohrmann E H. Clancy Behavior Scale[M]. Springer New York, 2013.

[23] Wang R, Chen F, Chen Z, et al. StudentLife: assessing mental health, academic performance and behavioral trends of college students using smartphones[C]// Proceedings of the 2014 ACM International Joint Conference on Pervasive and Ubiquitous Computing. ACM, 2014:3-14.

[24]Eagle N, Pentland A. Reality mining: sensing complex social systems[J]. Personal \& Ubiquitous Computing, 2006, 10(4):255-268.

[25] funf-open-sensing-framework. https://code.google.com/p/funf-open-sensing-framework/.

[26] Oliver P. John and Sanjay Srivastava. The Big Five trait taxonomy: History, measurement, and theoretical perspectives. In Lawrence A.Pervin and Oliver P. John, editors, Handbook of Personality: Theory and Research, pages 102-138. Guilford Press, New York, second edition, 1999.

[27]A. Rodriguez and A. Laio, "Clustering by fast search and find of density peaks," Science, vol. 344, no. 6191, pp. 1492-1496, 2014.

[28]Núñez J A, Cincotta P M, Wachlin F C. Information entropy[J]. Celestial Mechanics \& Dynamical Astronomy, 1996, 64(1-2):43-53. 